\title{
Extraction of Oleic Acid from Animals Oil and Its Anti-inflammatory Effect on Network Pharmacology
}

\author{
Jiuwang $\mathrm{Yu}^{1} \cdot \mathrm{Lu} \mathrm{Wang}^{1} \cdot$ Jiang $\mathrm{Ding}^{2} \cdot$ Lan $\mathrm{Wu}^{2}$ (D)
}

Received: 6 July 2020 / Accepted: 16 June 2021 / Published online: 19 August 2021

(C) The Author(s) 2021

\begin{abstract}
The purpose of this paper is to explore the possible mechanisms of anti-inflammatory and scar repair by Mongolian horse oil. We used TCM database and literature mining to collect active compounds of horse oil and used Swiss TargetPrediction and SuperPred server to find targets of compounds. Anti-inflammatory drug targets were collected through the CTD database. Go annotation of targets and KEGG pathway were enriched and analyzed through Metascape database platform. Molecular docking between active ingredients and targets was verified by AutoDock software. Metascape analysis revealed that the key candidate targets were significantly enriched in a number of pathways associated with inflammatory pathology. The results of molecular docking showed that oleic acid, a major component of animals oil, could influence the regulatory functions of TNF, NGF, IL6, IL1B, Jun, and CDK1. This suggests that animals oil can regulate the development of inflammation through its active ingredient, oleic acid, and can influence the expression of multiple signaling pathways, with theoretical endogenous interactions with TNF, NGF, IL6, IL1B, JUN, and CDK1 proteins.
\end{abstract}

Keywords Horse oil · Oleic acid · Network pharmacology Inflammation · TNF

\section{Introduction}

Daubing and rubbing therapy is to daub animal fat, yoghurt, milk wine and special medicine on the specific acupoints or parts of the human body, press and rub or massage to achieve the purpose of disease prevention and treatment. It is a kind of traditional external treatment combined with rub therapy and massage therapy. Mongolian medicine has a long history of rubbing therapy. In modern Mongolian medicine, daubing therapy is often used for severe insomnia, yellow water disease, anti-anxiety, chronic fatigue syndrome, hay disease, as well as for the

Jiuwang $\mathrm{Yu}$ and Lu Wang have equal contributions to the paper and are considered as co-first authors.

Lan Wu

wulan@immu.edu.cn

1 College of Pharmacy, Inner Mongolia Medical University, Hohhot, People's Republic of China

2 College of Mongolian Medical, Inner Mongolia Medical University, Hohhot 010059, Inner Mongolia, People's Republic of China treatment of disease, health care and so on (Kim and Jung 2013).

Horse oil is processed from the subcutaneous fat of horses. Horse oil has medicinal value, mild nature and sweet acid. It is one of the important folk medicines used to treat scald in Inner Mongolia. It has the effects of clearing heat and detoxification, detumescence and pain relief, moistening intestines and defecating stool, tonifying Chinese $\mathrm{Qi}$, moistening skin and generating muscles. It mainly treats the symptoms of deficiency of Chinese Qi, stomach ulcer, hemorrhoids, burn and scald, frostbite, chapped skin, aching limbs and nameless swelling and pain (Deak et al. 2018). According to the historical book compendium of Materia Medica, it is also effective in treating prolapse of uterus, hemoptysis, scabies, baldness and infantile chancre.

Horse oil has a good effect in the treatment of diseases, especially in the treatment of burns and scalds. According to previous studies, Mongolian medicine horse oil is rich in oleic acid, which has a good inhibitory effect on Grampositive bacteria and negative bacteria, but its deep mechanism in the treatment of burns and scalds and scar repair has not been reported (McDonald 2016). In this project, the extraction of oleic acid from horse oil is explored, and the target of its biological activity is found 
through network pharmacology, which provides the basis for the future drug development.

\section{Materials and Methods}

\subsection{Material Science}

Re-85z rotary evaporator, hh-s constant temperature water bath, yb-1a vacuum drying oven, zf1-ii ultraviolet analyzer, kq-250de numerical control ultrasonic cleaner, Agilent 1260 high-performance liquid chromatograph (Agilent, USA), and Agilent 1260 infinity evaporative light scattering detector (Agilent, USA) were used. Horse oil is purchased from the badger Duobao domestication farm in Ordos City. Column chromatography silica gel (100200200-300 mesh), thin-layer chromatography silica gel gf254 (Qingdao marine chemical plant), iodine (analytical pure, Tianjin Jinfeng Chemical Co., Ltd.), acetonitrile chromatography pure, American Oceanpak Alexander chemical), and other chemical reagents are analytical pure.

\subsection{Extraction of Oleic Acid from Horse Oil}

Take badger hypodermic horse oil and add organic solvent to extract it, heat it in water bath, after extracting for a period of time, extract and separate the supernatant and residue containing oil, collect the supernatant, use rotary evaporator to spin at $75{ }^{\circ} \mathrm{C}$ to separate the oil, clean the oil with anhydrous ethanol to obtain dry and pure horse oil, and weigh it. Determination of acid value of horse oil: refer to GB/T 5530-2005 determination of acid value and acidity of animal and vegetable oils; Determination of fatty acid composition of horse oil: refer to GB/t17376-2008 gas chromatography separation of fatty acid methyl esters of animal and vegetable oils. $100 \mathrm{ml}$ horse oil was separated and purified. 95\% ethanol was heated at $75^{\circ} \mathrm{C}$ and refluxed for 2 times, each time for $1 \mathrm{~h}$. The light yellow transparent oil liquid was obtained by combining the extract and concentrating the recovered ethanol under reduced pressure. $95 \%$ ethanol extraction part: four components were obtained by normal phase silica gel column chromatography and gradient elution of petroleum ether $\left(60-90{ }^{\circ} \mathrm{C}\right)$ ethyl acetate. The first component was separated and purified by normal phase preparation TLC. Non 95\% ethanol extraction part: repeatedly use the normal phase silica gel column chromatography separation, petroleum ether $\left(60-90{ }^{\circ} \mathrm{C}\right)$-dichloromethane, petroleum ether $\left(60-90{ }^{\circ} \mathrm{C}\right)$-ethyl acetate gradient elution, and normal phase preparation TLC purification to obtain oleic acid (Niu et al. 1995).

\subsection{Collection and Screening of Candidate Active Compounds in Horse Oil}

TCMSP (http://lsp.nwu.edu.cn/tcmsp.PHP) and ETCM (http://www.nrc.ac.cn:9090/ETCM/index.PHP/home/ index/) databases were searched to collect horse oil related active compounds ( $\mathrm{Ru}$ et al. 2014). TCMSP database screening criteria: oral bioavailability $(\mathrm{OB}) \geq 30 \%$ and drug similarity $(\mathrm{DL}) \geq 0.18$; the screening principle of ETCM database is five principles of Lipinski drugs (molecular weight is less than 500; the number of hydrogen bond donors is less than 5; the number of hydrogen bond receptors is less than 10 ; the partition coefficient of lipid and water is less than 5; and the number of rotatable bonds is not more than 10); the main blood components and blood metabolites are collected through literature retrieval, and the compounds with higher content and active compounds are reported. Through literature mining and using TCMSP and PubChem (https://PubChem.NCBI.NLM.NIH.Gov/), the molecular structure of each active compound was confirmed (Zhu et al. 2019).

\subsection{Prediction of Potential Targets}

Using the PubChem (https://pubchem.ncbi.nlm.nih.gov/) database, all compounds are converted into the standard canonical smiles format, and the smiles format file is imported into Swiss TargetPrediction (http://www.swis stargetprediction.ch/) and SuperPred website (http://predic tion.charite.de/), with the attribute of "home sapiens" to predict the target of compounds (Safhi et al. 2014). Online prediction platform of Swiss selects the target whose probability is $\geq 0.6$ in the prediction result for further analysis. SuperPred can predict the potential targets of unknown molecules by calculating the Tanimoto similarity between molecules and more than 300,000 known compounds in the server. The prediction results of Swiss TargetPrediction and SuperPred website are collected and deweighted, which are used as the prediction target of horse oil for further analysis.

\subsection{Collection of Inflammation Related Targets}

The targets with an inference score greater than or equal to 10 were selected from the CTD (http://ctd.mdibl.org/) database as the targets of anti-inflammatory drugs, and the database of inflammation related targets was established by literature mining. By comparing and analyzing the abovementioned target of horse oil interaction with inflammation related target, the prediction target with definite anti-inflammatory effect was summarized, and finally the target 
gene pool of horse oil anti-inflammatory effect was established (Wiegers et al. 2014).

\subsection{Protein and Gene Information Correction}

Because the retrieved targets may have irregular names or contain proteins or genes from different species, the target information needs to be standardized after each step of target collection. The specific methods are as follows: use uniprotkb search function in UniProt database, input protein name and define the species as human (Homo sapiens), correct all the retrieved proteins to their official names (official symbols) and extract the standard gene names, and obtain the correct target information through database retrieval and transformation (Shaik et al. 2016).

\subsection{Gene Analysis and Pathway Annotation}

The Metascape platform (http://metascape.org) is a gene annotation analysis database, which is used to analyze the biological processes and pathways of input genes. The antiinflammatory target of horse oil was input into the Metascape platform. After submission, the input species and analysis species were selected as "Hsapiens", with $p<0.01$ set. GO annotation analysis and KEGG pathway analysis were conducted on the target of horse oil, the results were saved, and the target points involved in each entry were sorted, and the top biological processes and pathways were screened (Zhou et al. 2019).

\subsection{Functional Attribution of Target Protein}

Metascape platform for gene annotation analysis can not only analyze the biological process and pathway enrichment of input genes, but also map the genes corresponding to horse oil regulatory target protein directly to the pathway. The pathway enriched by drug target is considered to be the drug regulatory pathway. The combined target pathway network of horse oil was used to analyze the common target of anti-inflammatory effect of horse oil, and to clarify the synergistic effect or superposition between drugs and pathways (Zhang et al. 2019a, b).

\subsection{Molecular Docking}

Using "burn" as the key word, we obtained the related target genes on the genecards platform, and sequenced them through the correlation score (Shen et al. 2019). Previous studies of our group showed that Jun, CDK1 and other genes play an important role in the process of early burn stimulation response. In this study, the key target and oleic acid were molecular docking. The 3D structure file of the protein was retrieved and downloaded by RCSB PDB (http://www.rcsb.org/pdb/home/home.do) database, and the $3 \mathrm{D}$ structure file of the active compound was downloaded by zinc database (http://zinc.docking.org/). Before docking, the energy of the ligand and the receptor molecule needed to be minimized, the water molecule of the receptor molecule (in PDB file) was deleted, and the polar hydrogen atom was added, Give charge, add magnetic field. All substructures within the radius of $0.65 \mathrm{~nm}$ were used as the active pocket of the binding site. Autodock molecular docking software (version 2.5) was used to connect oleic acid and receptor protein molecules to examine the binding mode and binding free energy of the compound with the corresponding target to determine the affinity between them (Basati et al. 2019) The technical route of the wholeresearch is shown in Figure 1.

\section{Results}

\subsection{Target Prediction}

According to Swiss TargetPrediction and SuperPred website, the target of horse oil active compound oleic acid was predicted, and the assumed target of oleic acid was determined based on the experimental results. According to the inference score, 101 known targets of inflammatory therapy in CTD database were selected to construct the database of inflammatory disease targets. The results with high expression abundance are shown in Table 1.

\subsection{Gene Function and Pathway Enrichment Analysis}

Online prediction platform of Swiss TargetPrediction selects the target whose probability is $\geq 0.6$ in the prediction result for further analysis. The potential targets of unknown molecules are predicted. The prediction results of the database are summarized and de-weighted, which is used as the prediction target of horse oil, so that further analysis can be made to obtain 6 disease treatment directions (Fig. 2A) GO annotation analysis and KEGG pathway analysis were conducted on the potential targets of horse oil anti-inflammatory disease by using Metascape platform, and the threshold value $p<0.01$ was set to screen the top GO annotation results and KEGG pathway. The results are shown in Fig. 2B. The results showed that there were 20 highly overlapping signaling pathways, among which KEGG pathway related to inflammation was selected for annotation, and genes corresponding to horse oil regulatory target protein were mapped directly to the pathway. The overlap between the analysis gene lists is only at the gene level, and the purple curve links the same gene, including the shared term level analysis, in which the 
Table 1 The results with high expression abundance

\begin{tabular}{lllll}
\hline Target & Common name & Uniprot ID & ChEMBL ID & Target class \\
\hline Fatty acid binding protein adipocyte & FABP4 & P15090 & CHEMBL2083 & Fatty acid binding protein family \\
Tumor necrosis factor & TNF & O00519 & CHEMBL2243 & Enzyme \\
Peroxisome proliferator-activated receptor gamma & PPARG & P37231 & CHEMBL235 & Nuclear receptor \\
Peroxisome proliferator-activated receptor alpha & PPARA & Q07869 & CHEMBL239 & Nuclear receptor \\
Nerve growth factor & NGF & O14746 & CHEMBL2916 & Enzyme \\
Fatty acid binding protein epidermal & FABP5 & Q01469 & CHEMBL3674 & Fatty acid binding protein family \\
Peroxisome proliferator-activated receptor delta & PPARD & Q03181 & CHEMBL3979 & Nuclear receptor \\
Interleukin 6 & IL6 & P07148 & CHEMBL5421 & Fatty acid binding protein family \\
Fatty acid binding protein muscle & FABP3 & P05413 & CHEMBL3344 & Fatty acid binding protein family \\
Acyl-CoA desaturase & SCD & O00767 & CHEMBL5555 & Enzyme \\
Protein-tyrosine phosphatase 1B & PTPN1 & P18031 & CHEMBL335 & Phosphatase \\
Interleukin 1B & IL1B & P17706 & CHEMBL3807 & Phosphatase \\
HMG-CoA reductase & HMGCR & P04035 & CHEMBL402 & Oxidoreductase \\
Cyclooxygenase-1 & PTGS1 & P23219 & CHEMBL221 & Oxidoreductase \\
Free fatty acid receptor 1 & FFAR1 & O14842 & CHEMBL4422 & Family A G protein-coupled receptor \\
\hline
\end{tabular}

Fig. 1 Technical route of antiinflammatory mechanism of horse oil based on the network pharmacology

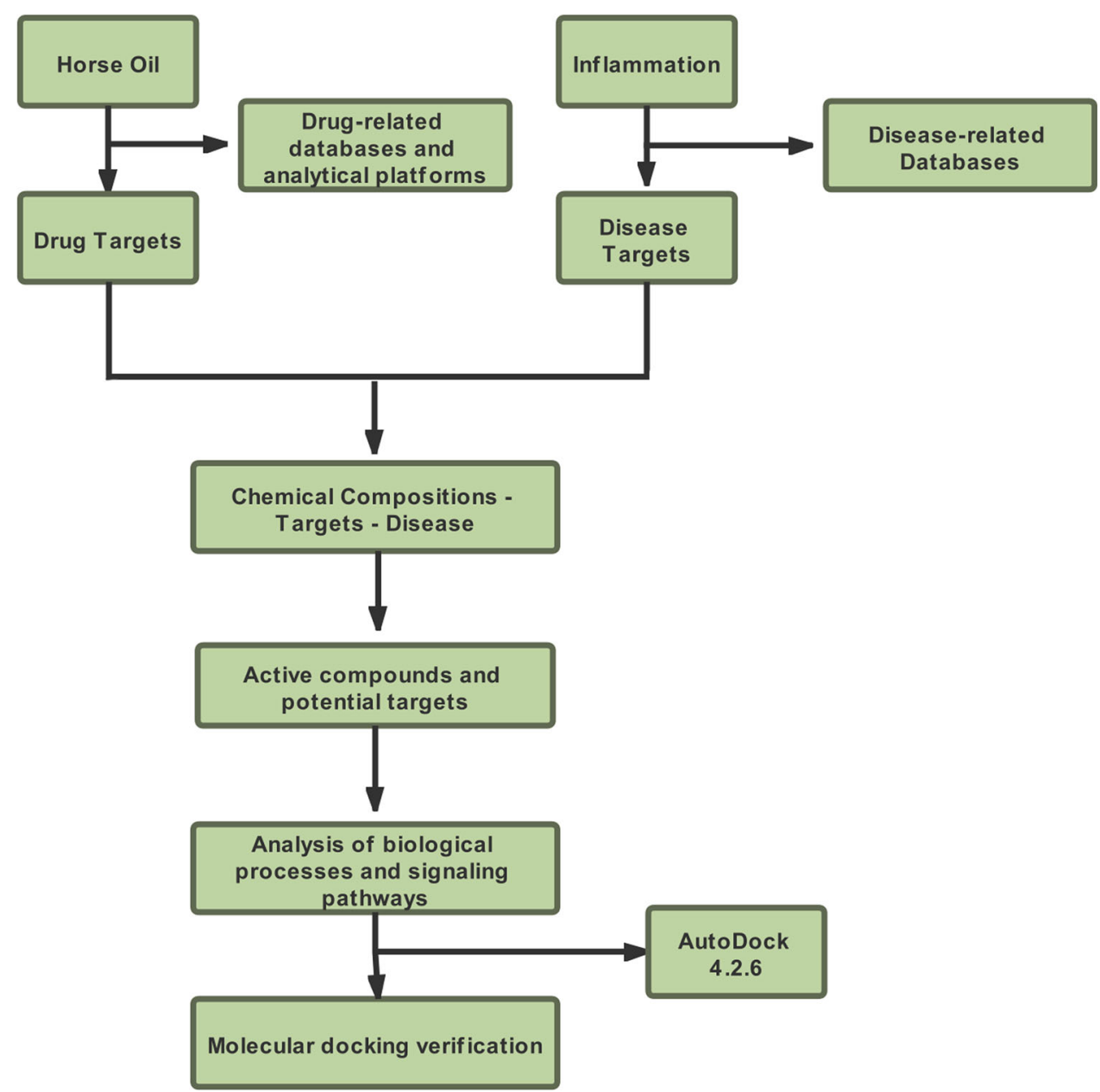


blue curve links belong to the same gene rich in ontology terms (Fig. 2C, D).

Through the enrichment analysis of KEGG pathway in the Metascape database, 20 pathways closely related to inflammation were found. In order to further determine the relationship between enrichment terms, kappa score was calculated as a measure of similarity between terms, and the enrichment term similarity network was constructed, as shown in Fig. 3A. Through the similarity between terms (kappa $>0.3$ ), the nodes are connected to form a network, and each node represents a enrichment term. The color of the node indicates the cluster to which the node belongs. It can be seen that the terms belonging to the same cluster are closer and more closely related to each other. The color of the node in Fig. 3B indicates its enrichment degree ( $p$ value). It can be seen that the more the number of genes included, the more significant the $p$ value.

\subsection{Functional Attribution of Target Protein}

Through the Metascape platform, all genes are connected and interacted with each other (Fig. 4A). Three different colors represent the module substructure recognized in the interaction network. The key genes corresponding to oleic acid regulatory target protein are directly mapped to pathway (Fig. 4B), and the pathway enriched by drug target is considered as drug regulatory pathway. It can be found that TNF, NGF, IL6, IL1B, JUN, CDK1 and other proteins are key targets related to inflammation (Fig. 4C).

\subsection{Molecular Docking Results}

Based on the above results, this study verified the top ten genes screened by Genecard (Table 2). The 3D structure is imported into Autodock, respectively, and butted with oleic acid. Their interaction patterns with the surrounding key
A

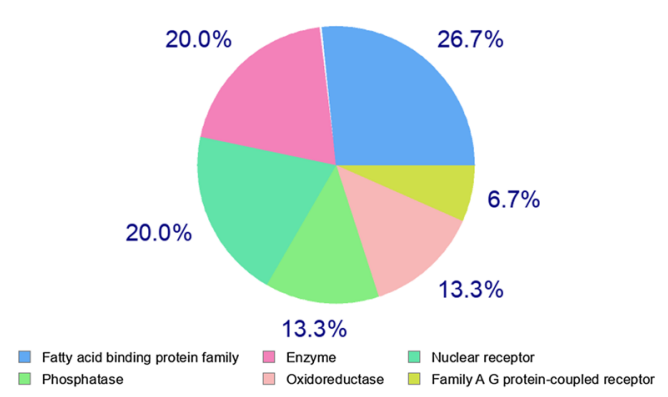

B

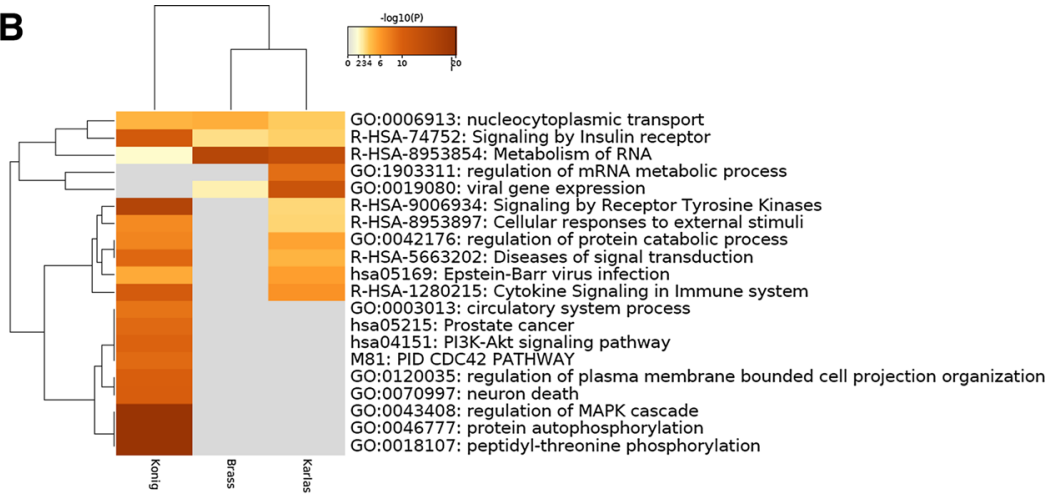

C

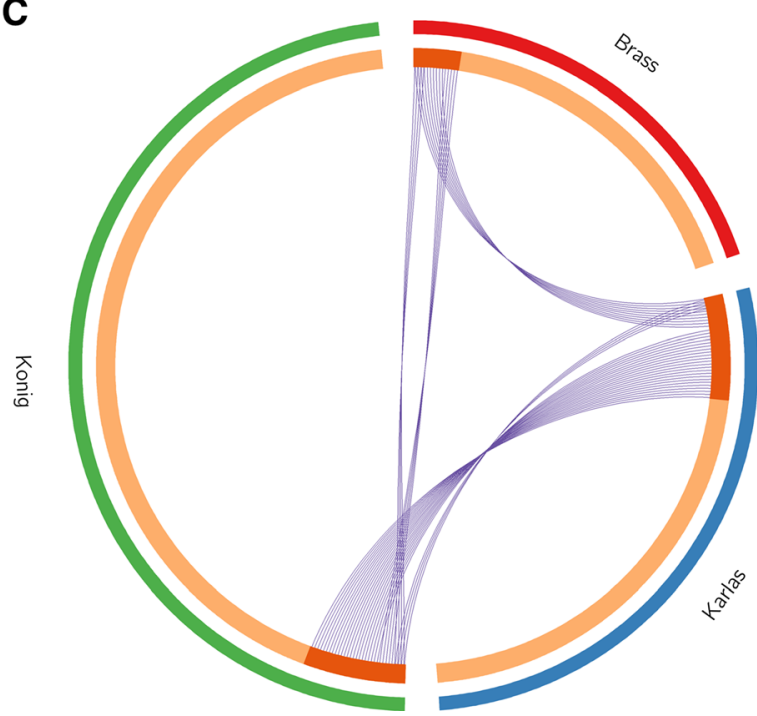

Fig. 2 A shows the six disease treatment directions predicted by Swiss TargetPrediction; B shows the top GO annotation results and KEGG pathway screened by Metascape platform; $\mathbf{C}$ shows the same gene linked by purple curve at gene level; $\mathbf{D}$ shows the shared term level analysis, where blue curve link belongs to the same gene rich in
D

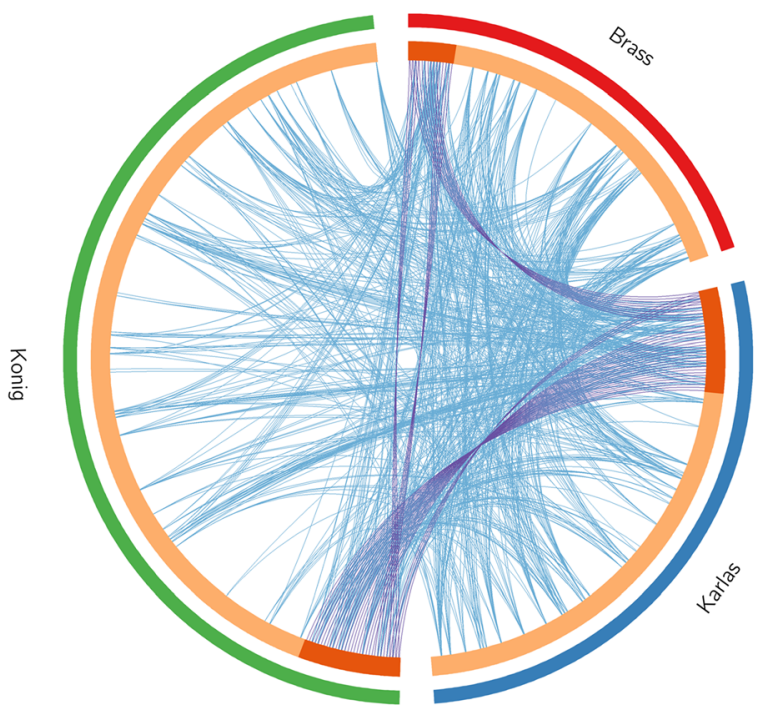

ontology terms. The inner circle represents the gene list, and the hits are arranged along the arc. Genes that hit multiple lists are colored in dark orange, while the only genes in the list are displayed in light orange 

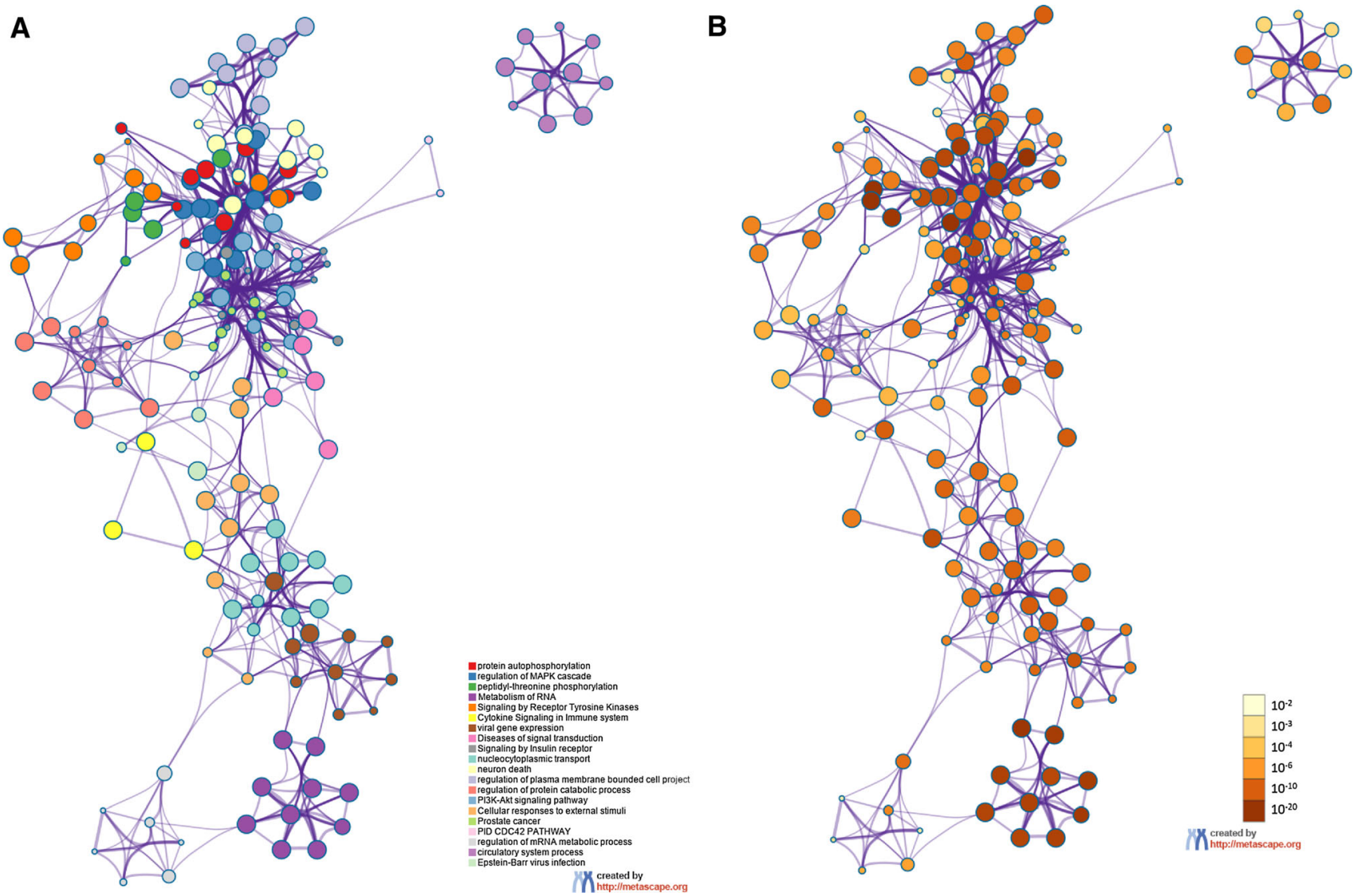

Fig. 3 A Network of similarity between terms; B node color indicates its enrichment degree

Table 2 Top 10 genes related from Genecards

\begin{tabular}{lll}
\hline NO & Gene symbol & Relevance score \\
\hline 1 & SCN9A & 34.39 \\
2 & TXNL4A & 34.39 \\
3 & TNF & 26.31 \\
4 & NGF & 25.80 \\
5 & IL6 & 25.59 \\
6 & IL1B & 22.59 \\
7 & CRP & 20.56 \\
8 & SCN10A & 19.89 \\
9 & JUN & 19.82 \\
10 & CDK1 & 19.46 \\
\hline
\end{tabular}

amino acids and their binding at the active site are shown in Fig. 5, and the energy values of the compounds shown by the docking results are shown in Table 3. Among them, SCN9A, TXN14A, CPR and SCN10A failed to retrieve the 3D structure of the protein. The results of molecular docking showed that oleic acid and corresponding protein were well docked, and the key amino acids around it mainly played a role in the form of hydrogen bond; combining with Table 3, the docking energy value was relatively small, which indicated that the compound can bind and act stably with the receptor protein (Fig. 5).

\section{Discussion}

Mongolian medicine daubing therapy has unique operation method, reliable effect and no side effects. Mongolian medicine application therapy is closely related to nomadic culture and life customs and keeps ancient medical traces. At that time, they were good at using horse oil, milk, camel milk, cream, marmot oil, milk wine, etc., commonly used in daily life as a medium for local application, and most of them used silver bowls and other special tools for Massage (Zhao et al. 2013). It is a popular health preserving method for the royal nobles. With the continuous enrichment of clinical application of Mongolian medicine rubbing therapy, it has developed into the treatment of soft tissue injury, rheumatoid arthritis, lumbago and leg pain and other clinical diseases. In recent years, horse oil rub therapy is widely concerned by the medical community and accepted by the majority of patients. Horse oil rub therapy does not need special medical equipment and is not limited 

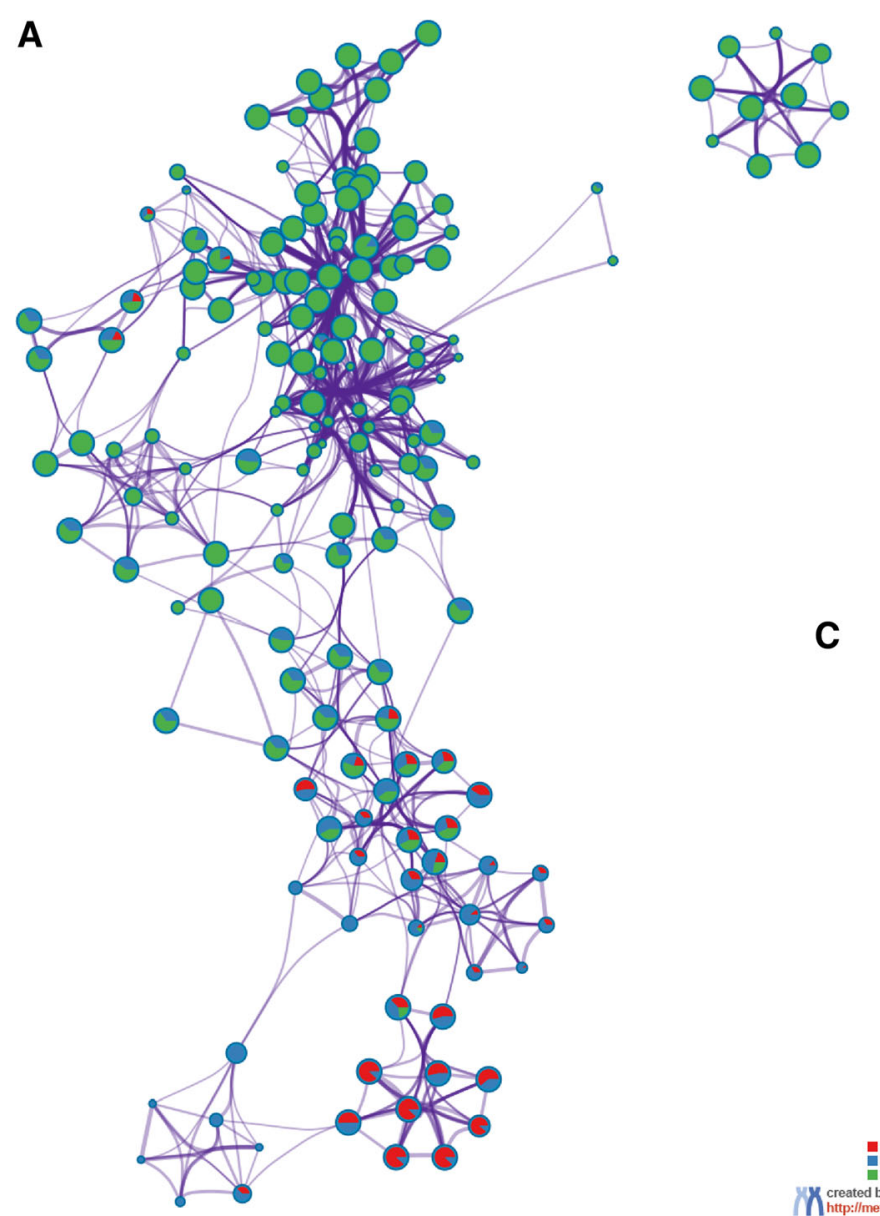

B

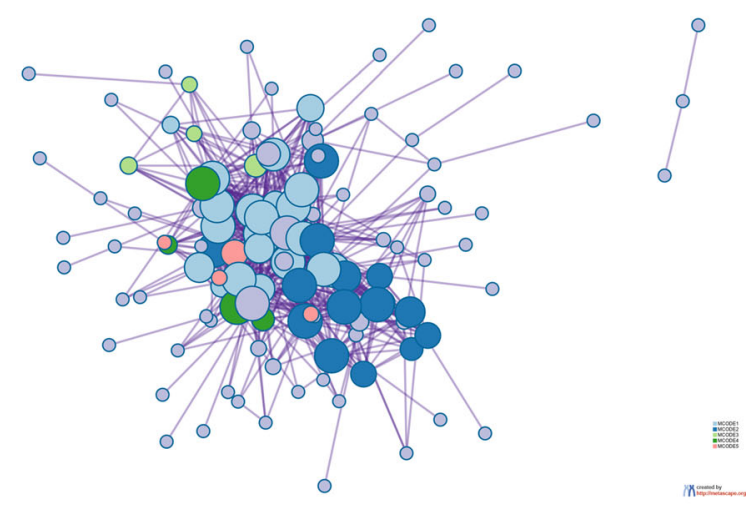

C
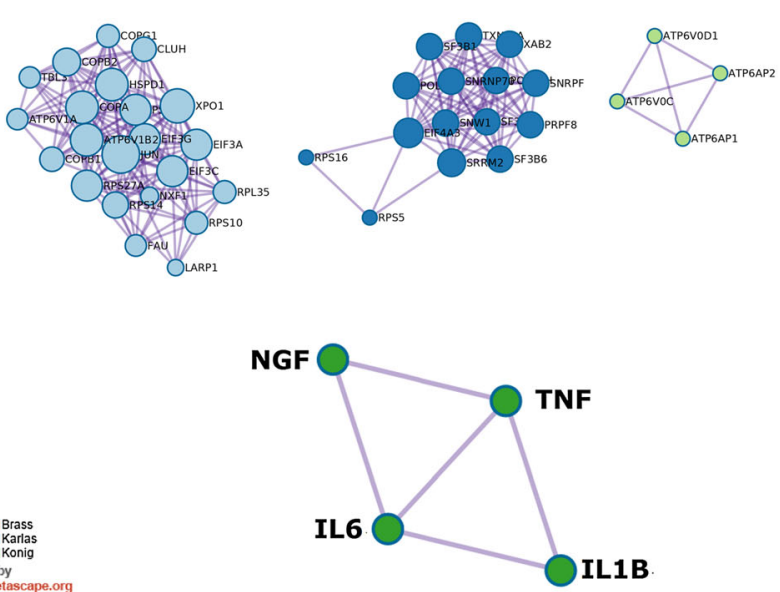

Fig. 4 A Rich term network represented by pie chart, in which pie is color coded according to the identity of gene list; $\mathbf{B}$ and $\mathbf{C}$ protein protein interaction network and code components identified in gene list

Table 3 Docking energy value

\begin{tabular}{llll}
\hline Chemical compound & Putative target & PDB ID & Binding energy \\
\hline Oleic acid & TNF & 6 RMJ & -0.21 \\
Oleic acid & NGF & 5JZ7 & -0.67 \\
Oleic acid & IL6 & $4 Z S 7$ & -1.75 \\
Oleic acid & IL1B & 1T4Q & -0.56 \\
Oleic acid & JUN & 1JUN & -0.46 \\
Oleic acid & CDK1 & $4 Y C 6$ & -0.18 \\
\hline
\end{tabular}

by the time, place and climate conditions. It can be carried out anytime and anywhere and is easy to learn and use, no inconvenience of taking medicine, no pain of acupuncture, no side effects, low-cost method of self-treatment, prevention and health care (Heddergott et al. 2015).

According to the basic theory of Mongolian medicine, horse oil rubbing therapy is closely related to the regulation of Qi and blood circulation. Horse oil massage therapy can make the human body full of Qi and blood, while full of Qi and blood can rejuvenate the human spirit. On the contrary, when Qi and blood are declining, the human health will be damaged and the functions of viscera and organs will be affected. Horse oil massage therapy can give all kinds of stimulation to the patient's body surface, regulate the movement of Qi and blood, unblock the tendons, and cure all kinds of diseases (Qiburi et al. 2020). In modern medicine, it is believed that the horse oil rubbing technique can contact the skin of patients, make the local focus warm, expand blood vessels, improve blood circulation, eliminate the apoptotic epithelial cells on the skin surface, increase the luster and elasticity of the skin, facilitate the secretion of sweat glands and sebaceous glands, and improve the respiratory and excretory functions of human skin. It can increase the oxygen demand of local skin tissue, increase the output of nitrogen and carbon dioxide, achieve the effect of pain and swelling reduction, promote skin metabolism, play the role of beauty, body-building, moistening skin, and thus play the role in health care, body-building and disease prevention. In clinical practice, modern research results have been integrated into the basic theory 
A

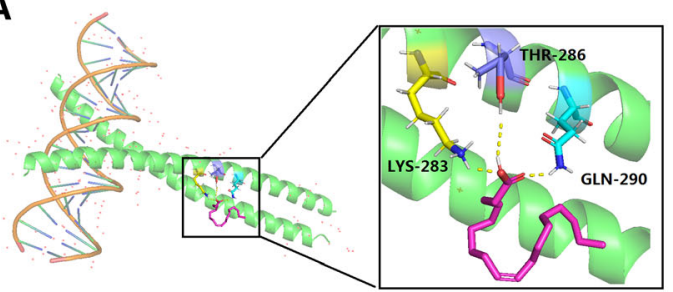

C
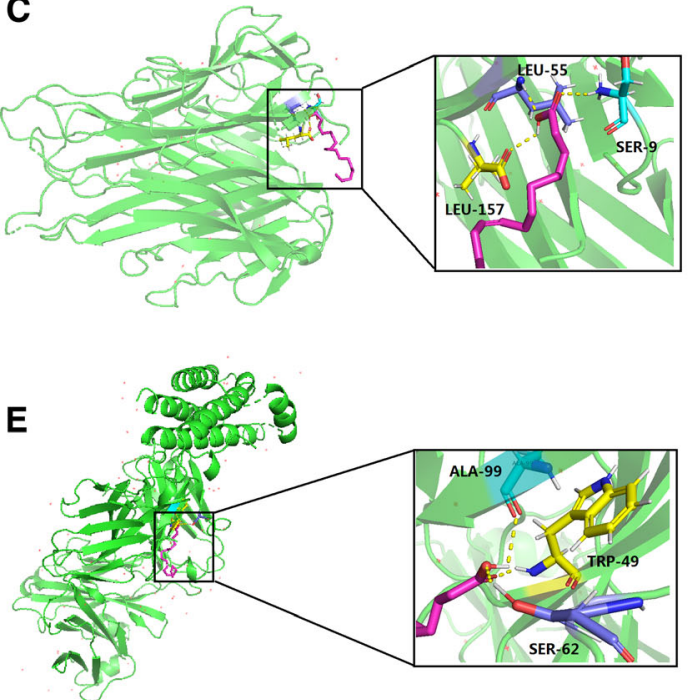

Fig. 5 A Site binding of active ingredient oleic acid to JUN; B site binding of active ingredient oleic acid to $\mathrm{CDK} 1 ; \mathbf{C}$ is the site binding of active ingredient oleic acid to TNF; $\mathbf{B}$ site binding of active

of horse oil therapy, and gratifying results have been achieved (Millán et al. 2007).

Horse oil is composed of fatty acids which are very similar to human skin fat and have strong penetration. Oleic acid can promote penetration by disturbing the lipid bilayer of cuticle and forming cracks. Oleic acid and linoleic acid can promote wound closure and inhibit the production of nitric oxide in wound tissue. The specific pharmacological mechanism and active components need further study. In the past, it was reported that oleic acid had a good inhibitory effect on Gram-positive and Gram-negative bacteria and had no sensitizing effect on the skin; the anti-inflammatory effect of oleic acid could prevent inflammation induced skin redness and swelling (Singh et al. 2020; Wang and Jiang 2020). Superoxide dismutase has the functions of anti-aging, anti-radiation, anti-inflammatory and anti-cancer. Oleic acid can be used as modifier to covalently modify the lysine on the surface of SOD. It has a long half-life, strong stability, no immunogenicity, and low molecular weight of oleic acid modified superoxide dismutase (Zhang et al. 2019a, b). In this project, through the network pharmacology method, it is proved that horse oil can regulate the development of inflammation
B
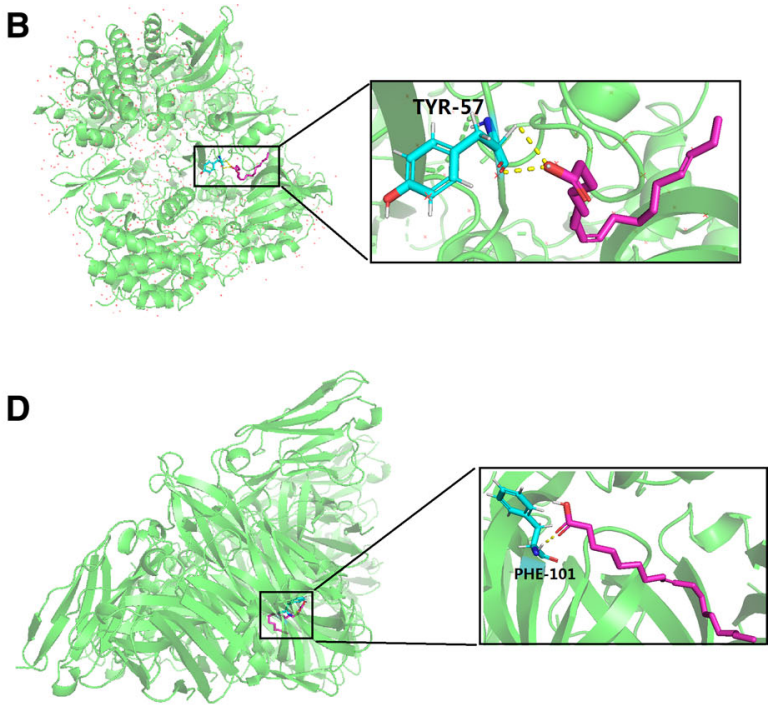

$\mathbf{F}$

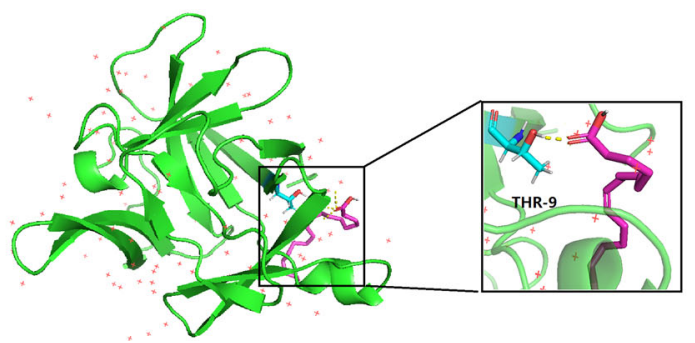

ingredient oleic acid to NGF; $\mathbf{D}$ site binding of active ingredient oleic acid to IL6; $\mathbf{E}$ and $\mathbf{F}$ site binding of active ingredient oleic acid to IL1B

through its active ingredient oleic acid and can affect the expression of multiple signal pathways, and there are endogenous interactions among TNF, NGF, IL6, IL1B, JUN and CDK1 proteins in theory (Song et al. 2012; Kany et al. 2018).

Horse oil can not only be used as medicine resources, but also as cosmetics and nutrition and health products resources. It has broad development prospects and needs further development and utilization. In the future, we can further verify the theoretical interaction protein obtained through network pharmacology.

Acknowledgements This research is funded by the following projects: Natural Science Foundation of Inner Mongolia (2020MS08046); The Major Science Foundation of Affiliated Hospital of Inner Mongolia Medical University; Natural Science Foundation of Inner Mongolia Autonomous Region (No. 2020MS08046, 2018LH08032 and 2019MS08110); Natural Science Foundation of Inner Mongolia Autonomous Region (2021ZD14); Inner Mongolia Autonomous Region "Prairie excellence" Project. The special Funds for the Local science and technology development of the central government (1280697693076717568), Inner Mongolia Autonomous Region Science and Technology Plan Project (1261196311571464192), "Western Light" Scholar Program of Chinese Academy of Sciences. 
Open Access This article is licensed under a Creative Commons Attribution 4.0 International License, which permits use, sharing, adaptation, distribution and reproduction in any medium or format, as long as you give appropriate credit to the original author(s) and the source, provide a link to the Creative Commons licence, and indicate if changes were made. The images or other third party material in this article are included in the article's Creative Commons licence, unless indicated otherwise in a credit line to the material. If material is not included in the article's Creative Commons licence and your intended use is not permitted by statutory regulation or exceeds the permitted use, you will need to obtain permission directly from the copyright holder. To view a copy of this licence, visit http://creativecommons. org/licenses/by/4.0/.

Author's Contributions JY designed and analyzed the data; JD provided technical assistance; LW provided intellectual input; JY supervised the overall study and advised on study design and data interpretation; LW wrote the manuscript; LW contributed to the revision of the article. All authors read and approved the final manuscript.

\section{References}

Basati G, Saffari-Chaleshtori J, Abbaszadeh S, Asadi-Samani M, Ashrafi-Dehkordi K (2019) Molecular dynamics mechanisms of the inhibitory effects of abemaciclib, hymenialdisine, and indirubin on CDK-6. Curr Drug Res Rev 11(2):135-141

Deak G, Mihalca A, Hirzmann J, Colella V, Tăbăran F, Cavalera M, Brudacă F, Bauer C, Ionică A, Alić A, Otranto D, Gherman C (2018) Validity of genus Perostrongylus Schlegel, 1934 with new data on Perostrongylus falciformis (Schlegel, 1933) in European badgers, Meles meles (Linnaeus, 1758): distribution, life-cycle and pathology. Parasit Vectors 11(1):568

Heddergott M, Frantz A, Jenrich J, Müller F (2015) Dissections of fresh skulls confirm low prevalence of Troglotrema acutum (Trematoda: Troglotrematidae) in German badgers (Meles meles). Parasitol Res 114(2):789-793

Kany S, Woschek M, Kneip N, Sturm R, Kalbitz M, Hanschen M, Relja B (2018) Simvastatin exerts anticancer effects in osteosarcoma cell lines via geranylgeranylation and c-Jun activation. Int J Oncol 52(4):1285-1294

Kim T, Jung S (2013) Mongolian traditional-style blood-letting therapy. J Alternat Complement Med (new York, N.y.) 19(12):921-924

McDonald J, Bailey T, Delahay R, McDonald R, Smith G, Hodgson D (2016) Demographic buffering and compensatory recruitment promotes the persistence of disease in a wildlife population. Ecol Lett 19(4):443-449

Millán J, Ruiz-Fons F, Márquez F, Viota M, López-Bao J, Paz Martín-Mateo M (2007) Ectoparasites of the endangered Iberian lynx Lynx pardinus and sympatric wild and domestic carnivores in Spain. Med Vet Entomol 21(3):248-254
Niu X, Liu L, Hu M, Chen X (1995) Some similarities in vascular effects of oleic acid and oxidized low-density lipoproteins on rabbit aorta. J Mol Cell Cardiol 27(1):531-539

Qiburi Q, Ganbold T, Bao Q, Da M, Aoqier A, Temuqile T, Baigude $\mathrm{H}$ (2020) Bioactive components of ethnomedicine Eerdun Wurile regulate the transcription of pro-inflammatory cytokines in microglia. J Ethnopharmacol 246:112241

Ru J, Li P, Wang J, Zhou W, Li B, Huang C, Li P, Guo Z, Tao W, Yang Y, Xu X, Li Y, Wang Y, Yang L (2014) TCMSP: a database of systems pharmacology for drug discovery from herbal medicines. J Cheminform 6:13

Safhi M, Alam M, Hussain S, Hakeem Siddiqui M, Khuwaja G, Jubran Khardali I, Al-Sanosi R, Islam F (2014) Cathinone, an active principle of Catha edulis, accelerates oxidative stress in the limbic area of swiss albino mice. J Ethnopharmacol 156:102-106

Shaik A, Thumma V, Kotha A, Kramadhati S, Pochampally J, Bandi S (2016) Molecular docking analysis of UniProtKB nitrate reductase enzyme with known natural flavonoids. Bioinformation 12(12):425-429

Shen H, Zhou Y, Zheng J, Zhu R (2019) "Multi-component-multitarget-multi-pathway" mechanism of Kuihua Hugan Tablets based on network pharmacology. China J Chin Materia Medica 44(7):1464-1474

Singh P, Gari M, Choudhury S, Shukla A, Gangwar N, Garg S (2020) Oleic acid prevents isoprenaline-induced cardiac injury: effects on cellular oxidative stress, inflammation and histopathological alterations. Cardiovasc Toxicol 20(1):28-48

Song H, Liu Z, Zheng L (2012) Expression of IFN- $\gamma$, IL- $1 \alpha$, NGF- $\beta$ and TNF- $\alpha$ during the development of cerebellar cortex of Western Anhui white goose. Zool Res 33(2):211-217

Wang J, Jiang W (2020) The effects of RKI-1447 in a mouse model of nonalcoholic fatty liver disease induced by a high-fat diet and in HepG2 human hepatocellular carcinoma cells treated with oleic acid. Med Sci Monit Int Med J Exp Clin Res 26:e919220

Wiegers T, Davis A, Mattingly C (2014) Web services-based textmining demonstrates broad impacts for interoperability and process simplification. Database J Biol Databases Curation 2014

Zhang J, Zhang S, Wang P, Guo N, Wang W, Yao L, Yang Q, Efferth T, Jiao J, Fu Y (2019a) Pinolenic acid ameliorates oleic acidinduced lipogenesis and oxidative stress via AMPK/SIRT1 signaling pathway in HepG2 cells. Eur J Pharmacol 861:172618

Zhang L, Lu D, Liu M, Zhang M, Peng Q (2019b) Identification and interaction analysis of key miRNAs in medullary thyroid carcinoma by bioinformatics analysis. Mol Med Rep 20(3):2316-2324

Zhao N, Wang M, Li X (2013) Fracture healing with osteopathy in traditional Mongolian medicine. J Traditional 33(1):130-133

Zhou Y, Zhou B, Pache L, Chang M, Khodabakhshi A, Tanaseichuk O, Benner C, Chanda S (2019) Metascape provides a biologistoriented resource for the analysis of systems-level datasets. Nat Commun 10(1):1523

Zhu N, Hou J, Ma G, Liu J (2019) Network pharmacology identifies the mechanisms of action of shaoyao Gancao decoction in the treatment of osteoarthritis. Med Sci Monit Int Med J Exp Clin Res 25:6051-6073 